\title{
Development and validation of a predictive model to determine the level of care in patients confirmed with COVID-19
}

Anh Nguyet Diep, Allison Gilbert, Claude Saegerman, Marjorie Gangolf, Vincent D'Orio, Alexandre Ghuysen \& Anne-Françoise Donneau

To cite this article: Anh Nguyet Diep, Allison Gilbert, Claude Saegerman, Marjorie Gangolf, Vincent D'Orio, Alexandre Ghuysen \& Anne-Françoise Donneau (2021): Development and validation of a predictive model to determine the level of care in patients confirmed with COVID-19, Infectious Diseases, DOI: 10.1080/23744235.2021.1903548

To link to this article: https://doi.org/10.1080/23744235.2021.1903548

\section{Published online: 01 Apr 2021.}

Submit your article to this journal $\pi$

Q View related articles $\sqsubset$

View Crossmark data $\nearrow$ 


\title{
Development and validation of a predictive model to determine the level of care in patients confirmed with COVID-19
}

\author{
Anh Nguyet Diep ${ }^{a, b, c} \mathbb{D}^{\mathrm{D}}$, Allison Gilbert ${ }^{\mathrm{d}}$, Claude Saegerman $^{\mathrm{e}}$, Marjorie Gangolf ${ }^{\mathrm{f}}$, Vincent D'Orio ${ }^{\mathrm{d}}$, \\ Alexandre Ghuysen ${ }^{\mathrm{a}, \mathrm{d} *}$ and Anne-Françoise Donneau ${ }^{\mathrm{a}, \mathrm{b} *}$

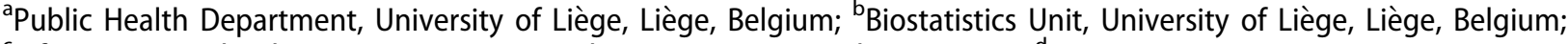

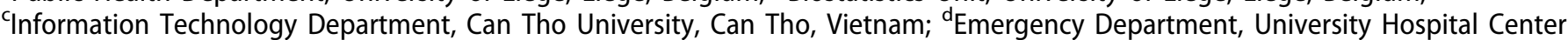 \\ of Liège, University of Liège, Liège, Belgium; ${ }^{e}$ Fundamental and Applied Research for Animal and Health (FARAH) Center, \\ University of Liège, Liège, Belgium; 'Department of Medico-Economic Information, University of Liège, Liège, Belgium
}

\section{ABSTRACT}

Background: The COVID-19 pandemic has imposed significant challenges on hospital capacity. While mitigating unnecessary crowding in hospitals is favourable to reduce viral transmission, it is more important to prevent readmissions with impaired clinical status due to initially inappropriate level of care. A validated predictive tool to assist clinical decisions for patient triage and facilitate remote stratification is of critical importance.

Methods: We conducted a retrospective study in patients with confirmed COVID-19 stratified into two levels of care, namely ambulatory care and hospitalization. Data on socio-demographics, clinical symptoms, and comorbidities were collected during the first $(N=571)$ and second waves $(N=174)$ of the pandemic in Belgium (2 March to 6 December 2020). Univariate and multivariate logistic regressions were performed to build and validate the prediction model.

Results: Significant predictors of hospitalization were old age $(\mathrm{OR}=1.08,95 \% \mathrm{Cl}: 1.06-1.10)$, male gender $(\mathrm{OR}=4.41$, 95\%Cl: 2.58-7.52), dyspnoea (OR 6.11, 95\%Cl: 3.58-10.45), dry cough (OR 2.89, 95\%Cl: 1.54-5.41), wet cough (OR 4.62, $95 \% \mathrm{Cl}: 1.93-11.06)$, hypertension (OR 2.20,95\%Cl: 1.17-4.16) and renal failure (OR 5.39, 95\%Cl: 1.00-29.00). Rhinorrhea (OR $0.43,95 \% \mathrm{Cl}: 0.24-0.79$ ) and headache (OR $0.36,95 \% \mathrm{Cl}: 0.20-0.65)$ were negatively associated with hospitalization. A receiver operating characteristic (ROC) curve was constructed and the area under the ROC curve was 0.931 (95\% Cl: 0.910-0.953) for the prediction model (first wave) and 0.895 (95\% Cl: 0.833-0.957) for the validated dataset (second wave). Conclusion: With a good discriminating power, the prediction model might identify patients who require ambulatory care or hospitalization and support clinical decisions by Emergency Department staff and general practitioners.

\section{KEYWORDS}

COVID-19

clinical symptoms

comorbidities

patient triage

level of care

\section{ARTICLE HISTORY}

Received 15 December 2020

Revised 9 March 2021

Accepted 10 March 2021

\section{CONTACT}

Anh Nguyet Diep

@anhnguyet.diep@uliege.be

*Both authors contributed equally to this work.

(C) 2021 Society for Scandinavian Journal of Infectious Diseases 


\section{Introduction}

Since the COVID-19 pandemic was recognized by the World Health Organization on 11 March 2020, the pandemic has challenged healthcare systems worldwide. To date (15 December 2020), 73,179,330 confirmed cases and 1,545,824 fatalities of COVID-19 have been reported in the world [1], exposing many countries to huge financial loss and fatalities. In this regard, Belgium, together with Peru and Italy, has shared unfortunate high death rates ( 1,572 per million inhabitants) [2].

Healthcare systems in these countries have experienced shortages of hospital beds and over-capacity challenges. Policymakers, healthcare professionals, and hospital management have sometimes worked with uncertainties when lack of intensive care unit (ICU) beds was expected within days.

Due to the complex nature of COVID-19 and lack of specific symptoms, patients with other infections may have similar symptoms. Many infected patients remain pauci- or asymptomatic but cause increased risk of viral transmission when they are admitted in hospitals or are in contact with vulnerable individuals such as the elderly, especially in crowded hospitals [3].

Together with strategies to reduce viral transmission such as physical distancing, wearing facemasks, and closing down schools and businesses, triage procedures in the Emergency Department have been widely used to identify COVID-19 patients, and to evaluate the need for in-hospital care. A predictive tool that might help stratify patients with suspected COVID-19 into different levels of care is of critical importance to reduce the pressure on hospitals and the risk of nosocomial spread. To be useful in the Emergency Department and assist clinicians in daily practice and ensure patient safety, such a tool should be simple, easy to use, and based on immediately accessible information.

Symptoms of COVID-19 range from flu-like symptoms such as fever, cough, and headache to more severe manifestations such as dyspnoea, myalgia, or anosmia [4]. Previous studies indicated that the risk of COVID-19 and hospitalization was associated with one or more comorbidities such as hypertension, heart disease, diabetes, renal failure, or pulmonary disease [5-7]. Moreover, old age and male gender, in the presence of comorbidities, are risk factors for severe complications and mortality [8].

To date, few studies have focussed on stratification of patients with confirmed COVID-19 at arrival to hospital based on socio-demographics, clinical symptoms, and comorbidities. Some studies examined either patient characteristics only or one type of comorbidity such as hypertension and had limited sample sizes $(n<200)$ $[3,9,10]$. However, as discussed by Wynants et al. [11], most prediction models for COVID-19 or outcomes were validated using internal validation approaches, such as dividing the dataset into training and testing datasets, or using a small or less representative external sample as in the study by Ryan et al. [3].

These concerns motivated us to design the present study using an original statistical model to identify the hospitalization risk for patients with confirmed COVID19. This model was developed and validated using data collected during the first and the second waves of the pandemic at the University Hospital Centre of Liège, Belgium. We aimed to develop and validate a prediction tool to support clinical decisions in patient triage and to facilitate remote stratification when in-person evaluations must be restricted during crisis management.

\section{Materials and methods}

\section{Research context}

The study was conducted during the first and second waves of the SARS-Cov-2 outbreak in Belgium at the two Emergency Department triage centres of the University Hospital Centre of Liège. According to the strategies implemented by the Belgian government, the first wave period from 2 March 2020 to 15 June 2020 was divided into three stages [4]. The pre-lockdown stage from 2 March to 17 March marked the beginning of the pandemic in Belgium. The lockdown stage from 18 March to 4 May was characterized by the implementation of strict measures including closing non-essential shops, travel restrictions, and contact prohibition among friends and family members. In the post-lockdown stage from 4 May to 15 June, restrictions were gradually lifted, and businesses resumed, hospital activities were reorganized, and in-person meetings and contacts were possible. 18 June 2020 marked the second wave of the pandemic in Belgium. Up to the time of the present study, i.e. 6 December 2020, lockdown measures have been implemented and are expected to be relaxed in February 2021 after a thorough evaluation by the Belgian National Security Council.

During the study period, patients presenting at the two centres were triaged based on a non-invasive clinical triage scale, which included 11 symptoms ranging from flu-like to more severe conditions (see further below). Patients were directed to the COVID centre if 
they had one of these symptoms. During the first wave, socio-demographic data, hospital administrative data, clinical symptoms, and comorbidities of 4,489 patients aged 16 years or older were entered into the database. After initial evaluation, 2,735 patients were tested for SARS-Cov-2. In the analysis, we included 571 out of 628 patients who were confirmed with COVID-19 based on real-time polymerase chain reaction (RT-PCR) analysis of nasopharyngeal swabs [4] and whose medical records were complete. In hospitalized patients, RT-PCR was done more than once if chest computed tomography (CT) showed findings compatible with COVID-19. One hundred and seventy-four patients with confirmed COVID-19 were eligible for inclusion in the second wave dataset.

\section{Variables}

Three categories of independent variables were collected. Socio-demographic variables included age and gender. Eleven clinical symptoms were recorded: dyspnoea, chest pain, rhinorrhea, sore throat, dry cough, wet cough, diarrhoea, headache, myalgia, fever, and anosmia. Seven types of comorbidities were registered: arterial hypertension, diabetes, cardiac disease, immunosuppression, pulmonary disease, cancer, and chronic renal failure. The outcome variables referred to the level of care patients received after testing positive for COVID-19. These included ambulatory care (discharged home the same day) and hospitalization (admission to a hospital unit for further treatment). If a patient was first discharged but admitted to the hospital a few days later, he/she was registered in the hospitalization group.

\section{Data analysis methods}

First of all, descriptive statistics were performed. Quantitative variables were described by median and interquartile ranges (IQR) and qualitative variables by frequencies and percentages. Comparisons of sociodemographics, clinical symptoms, and different types of comorbidities were conducted across two categories of patients, namely ambulatory care and hospitalization. Mann-Whitney $U$ test was used for quantitative variables and Fisher's exact test or $\chi^{2}$ test for qualitative variables.

To assess the likelihood of hospitalization, univariate binary logistic regression was done to examine the association between socio-demographics, clinical symptoms, comorbidities, and the level of care. Variables with $p$ value $<.05$ were included in the multivariate analysis, using backward elimination. Accordingly, variables with $p$-values $=>.05$ were not included. The final model was selected by the likelihood-ratio test which compared the simplified and the complex models. The results are presented as odds ratios (ORs) with $95 \%$ confidence intervals (Cls). The model fit was evaluated by Hosmer-Lemeshow test, $R^{2}$ (Cox-Snell and Nagelkerke, respectively), and the area under the curve (AUC) of the receiver operating characteristic (ROC) curve.

Sensitivity analysis was performed to test the robustness of the prediction function by comparing the AUCs obtained with the full dataset and with the sub-datasets when removing data from one of the three stages. The predictive values of the significant variables were also calculated after removing age and gender. Additionally, we tested if the results of the multivariate model changed if variables with $p<.10, p<.15$, and $p<.20$ in univariate analysis were included in the model.

Finally, the model(s) was validated using the dataset obtained from the second wave in which the $95 \% \mathrm{Cl}$ of the AUC-ROC was calculated using bootstrapping on 2000 bootstrapped samples. Furthermore, risk score models were developed using the ORs from the final logistic regression model to facilitate the practical use of the tool. Before the risk model was built, Youden's index, i.e. the sum of sensitivity and specificity minus one, was used together with the ROC curve, to determine the best cut-off value for age. Subsequently, the AUC-ROC was calculated for each risk score model, to select the most explanatory model. The analyses were performed in SPSS v.26 and the pROC package in R [12].

The study design was approved in principle by the Ethical Committee of the University of Liege before it was started and the official approval with reference number 2021-013 was received on 26 January 2021. Informed consent was exempted by the Ethical Committee for this type of study due to the anonymity of data retrieval.

\section{Results}

\section{Participants}

In the first wave, patients aged 50-59 years constituted the largest group $(n=100,17.5 \%)$, followed by patients older than 79 years $(n=97,17.0 \%)$ and 60-69years ( $n=16.6 \%)$. The median age of all 571 patients was 58 years, [IQR 40-73]. The number of female patients ( $n=293,51.3 \%$ ) was slightly higher than that of male patients $(n=278,48.7 \%)$. Most patients were from Belgium ( $n=565,98.9 \%)$. In the lockdown stage from 18 
Table 1. Descriptive statistics of socio-demographics, transport, and the required level of care $(N=571)$.

\begin{tabular}{|c|c|c|c|}
\hline Variables & Categories & First wave, $N(\%)$ & Second wave, $N(\%)$ \\
\hline & $16-19$ & $0(0.00)$ & $5(2.87)$ \\
\hline \multirow[t]{8}{*}{ Age group } & $20-29$ & $47(8.23)$ & $13(7.47)$ \\
\hline & $30-39$ & $89(15.59)$ & $14(8.05)$ \\
\hline & $40-49$ & $65(11.38)$ & $21(12.07)$ \\
\hline & $50-59$ & $100(17.51)$ & $33(18.97)$ \\
\hline & $60-69$ & $95(16.64)$ & 37 (21.26) \\
\hline & $70-79$ & $78(13.66)$ & $37(21.26)$ \\
\hline & $>79$ & 97 (16.99) & $14(8.05)$ \\
\hline & & $\begin{array}{c}\text { Median }=58 \\
\text { IQR }[40-73]\end{array}$ & $\begin{array}{l}\text { Median }=60.5 \\
\quad \text { IQR }[43-72]\end{array}$ \\
\hline \multirow[t]{2}{*}{ Gender } & Male & $278(48.69)$ & $100(57.47)$ \\
\hline & Female & $293(51.31)$ & $74(42.53)$ \\
\hline \multirow[t]{3}{*}{ Country } & Belgium & 565 (98.95) & $171(98.28)$ \\
\hline & France & $3(0.53)$ & $3(1.72)$ \\
\hline & Luxembourg & $3(0.53)$ & $0(0.00)$ \\
\hline \multirow[t]{6}{*}{ Period } & Wave 1 & & \\
\hline & Pre-lockdown stage (2 March-17 March) & $56(9.81)$ & \\
\hline & Lockdown stage (18 March-3 May) & $496(86.87)$ & \\
\hline & Post-lockdown stage (4 May-15 June) & 19 (3.33) & \\
\hline & Wave 2 & & \\
\hline & From 18 June to 6 December 2020 & & $174(100.00)$ \\
\hline \multirow[t]{2}{*}{ Level of care required } & Ambulatory care & $232(40.63)$ & $76(43.68)$ \\
\hline & Hospitalization & 339 (59.37) & $98(56.32)$ \\
\hline
\end{tabular}

March 2020 to 3 May 2020, the highest number of patients arrived at the two triage centres $(n=496$, $86.9 \%)$. Roughly two-thirds of the visits were self-referrals $(n=391,68.5 \%), 144(25.2 \%)$ visits required an ambulance, and 27 (4.7\%) needed doctor-led emergency medical services (EMS). After consultation and diagnosis, 232 (40.6\%) patients with confirmed COVID-19 were sent home and managed in ambulatory care, whereas 339 (59.4\%) were admitted to the hospital.

In the second wave, roughly half of the patients were above 60 years of age, with a median of 60.50 years [IQR 43-72]. Male patients accounted for $57.5 \%$ and most patients were from Belgium ( $n=171,98.3 \%)$. Hospital care was required in $56.3 \%(n=98)$ of patients and $43.7 \%(n=76)$ received ambulatory care.

Using Bonferroni correction, there was no significant difference in age between patients in wave 1 and wave $2(p=.820)$. Pearson chi-square test revealed a non-significant difference in the observed numbers of male and female patients $(p=.084)$ in both waves. The level of care did not differ significantly between the two $(p=.950)$.

The results are presented in Table 1.

\section{Socio-demographics, clinical symptoms, and comorbidities of patients with confirmed COVID-19}

Table 2 presents the results obtained from the Mann-Whitey, chi-square tests, and univariate logistic regression, in which patients were categorized into ambulatory care and hospitalization using data from the first wave.

Patients who were hospitalized were older than those in ambulatory care with a median age of 69 years [IQR 58-81] and 39years [IQR 32-53], respectively, OR 1.10 (95\%Cl: 1.08-1.12). More males $(n=206,60.8 \%)$ than females $(n=133,39.2 \%)$ were hospitalized, OR 3.44 (95\%Cl: 2.42-4.90).

There were significant differences in symptomatology between patients in ambulatory care and hospitalized ones. Dyspnoea $(p<.001)$ and wet cough $(p<.05)$ were more frequently reported by hospitalized patients, OR 5.57 (95\%Cl: 3.86-8.04) and OR 1.68 (95\%Cl: 1.01-2.80), respectively. Patients in ambulatory care more often reported rhinorrhea ( $p<.001$, OR $0.19,95 \% \mathrm{Cl}: 0.13-0.28)$, sore throat $(p<.001$, OR $0.28,95 \% \mathrm{Cl}: 0.18-0.42)$, dry cough $(p<.05$, OR 0.71, 95\%Cl: 0.51-0.99), headache ( $p<.001$, OR 0.15, 95\% $\mathrm{Cl}$ : 0.10-0.22), and myalgia ( $p<.001$, OR $0.24,95 \% \mathrm{Cl}$ : 0.17-0.34). There were no significant differences for chest pain, diarrhoea, fever, and anosmia.

Hospitalized patients were more likely to have hypertension ( $p<.001$, OR 6.67, 95\%Cl: 4.21-10.58), diabetes $(p<.001$, OR 3.84, 95\%Cl: 2.17-6.77), cardiac disease ( $p<.001$, OR 5.22, 95\%Cl: 3.02-9.01), cancer $(p=.01$, OR $3.76,95 \% \mathrm{Cl}: 1.28-11.11)$, and renal failure $(p<.001, \mathrm{OR}$ 9.95, 95\%Cl: 2.34-42.27). There were no significant differences in immune suppression and pulmonary disease between the two groups. 
Table 2. Group comparison and results from univariate binary logistic regression regarding the probability of hospitalization vs. ambulance care $(N=571)$.

\begin{tabular}{|c|c|c|c|c|}
\hline Variables & Ambulatory care $(n=232)$ & Hospitalization $(n=339)$ & $p$ Value $^{c}$ & OR $(95 \% \mathrm{Cl})$ \\
\hline \multicolumn{5}{|l|}{ Socio-demographics } \\
\hline Age & 39 [IQR: 32-53] & 69 [IQR: 58-81] & $<.001$ & $1.10(1.08-1.12)$ \\
\hline \multicolumn{5}{|l|}{ Gender } \\
\hline Male & $72(31.03)$ & $206(60.77)$ & $<.001$ & $3.44(2.42-4.90)$ \\
\hline Female & 160 (68.97) & $133(39.23)$ & & \\
\hline \multicolumn{5}{|l|}{ Clinical symptoms ${ }^{\mathrm{a}, \mathrm{b}}$} \\
\hline Dyspnoea & $65(28.02)$ & $232(68.44)$ & $<.001$ & $5.57(3.86-8.04)$ \\
\hline Chest pain & $47(20.26)$ & $63(18.58)$ & 0.618 & $0.9(0.59-1.37)$ \\
\hline Rhinorrhea & $120(51.72)$ & $57(16.81)$ & $<.001$ & $0.19(0.13-0.28)$ \\
\hline Sore throat & 77 (33.19) & $41(12.09)$ & $<.001$ & $0.28(0.18-0.42)$ \\
\hline Dry cough & $134(57.76)$ & $167(49.26)$ & .046 & $0.71(0.51-0.99)$ \\
\hline Wet cough & $24(10.34)$ & $55(16.22)$ & .046 & $1.68(1.01-2.8)$ \\
\hline Diarrhoea & $46(19.83)$ & $83(24.48)$ & .191 & $1.31(0.87-1.97)$ \\
\hline Headache & $156(67.24)$ & $79(23.3)$ & $<.001$ & $0.15(0.1-0.22)$ \\
\hline Myalgia & $140(60.34)$ & $90(26.55)$ & $<.001$ & $0.24(0.17-0.34)$ \\
\hline Fever & $145(62.5)$ & $225(66.37)$ & .341 & $1.18(0.84-1.68)$ \\
\hline Anosmia & $6(2.59)$ & $3(0.88)$ & .109 & $0.34(0.08-1.36)$ \\
\hline \multicolumn{5}{|l|}{ Comorbidities $^{\mathrm{a}, \mathrm{b}}$} \\
\hline Hypertension & $26(11.21)$ & $155(45.72)$ & $<.001$ & $6.67(4.21-10.58)$ \\
\hline Diabetes & $16(6.9)$ & $75(22.12)$ & $<.001$ & $3.84(2.17-6.77)$ \\
\hline Cardiac disease & $17(7.33)$ & $99(29.2)$ & $<.001$ & $5.22(3.02-9.01)$ \\
\hline Immunosuppression & $12(5.17)$ & $15(4.44)$ & .685 & $0.85(0.39-1.85)$ \\
\hline Pulmonary disease & $26(11.21)$ & $46(13.57)$ & .404 & $1.24(0.75-2.08)$ \\
\hline Cancer & $4(1.72)$ & $21(6.19)$ & .010 & $3.76(1.28-11.11)$ \\
\hline Renal failure & $2(0.86)$ & $27(7.96)$ & $<.001$ & $9.95(2.34-42.27)$ \\
\hline
\end{tabular}

${ }^{a}$ The presence of trait is presented; ${ }^{b}$ Referenced category $=$ No; ${ }^{c} p$-value was for Mann-Whitney $U$ test of age differences and Pearson chisquare test of association for all qualitative variables.

\section{Predictors of hospitalization in patients with confirmed COVID-19: Multivariate logistic regression results}

Based on the results obtained from the univariate analysis, age, gender, dyspnoea, rhinorrhea, dry cough, wet cough, sore throat, headache, myalgia, hypertension, diabetes, cardiac disease, cancer, and renal failures were entered into the multivariate regression model as predictors.

The results revealed that the model was calibrated (Hosmer-Lemeshow test $\chi^{2}(8)=7.88, p=.445$ ). Significant predictors of being admitted to the hospital
Rhinorrhea (OR 0.43, 95\%Cl: 0.24-0.79) and headache (OR $0.36,95 \% \mathrm{Cl}: 0.20-0.65$ ) were negatively associated with the odds of hospitalization. Altogether, the model (model 1) accounted for an $R^{2}=0.504$ (Cox-Snell) and $R^{2}=0.680$ (Nagelkerke) with an AUC of $0.931(95 \% \mathrm{Cl}$ : 0.910-0.953), and standard error (SE) of 0.011, using the first wave dataset. The results are presented in Table 3 and the AUC is shown in Figure 1.

Using the significant predictors, a predictive function Equation (1) for the probability of hospitalization is derived as follows.

\footnotetext{
$p($ hospitalization $)=$

$\mathrm{e}^{-4.33+0.08 \mathrm{x}(\text { age })+1.48 \mathrm{x}(\text { male })+1.81 \mathrm{x}(\text { dyspnea })-0.84 \mathrm{x}(\text { rhinorrhea }+1.06 \mathrm{x}(\text { dry cough })+1.53 \mathrm{x}(\text { wet cough })-1.01 \mathrm{x}(\text { headache })+0.79 \mathrm{x}(\text { hypertension })+1.68 \mathrm{x}(\text { renal failure })}$

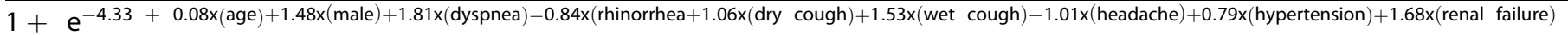

were old age (OR 1.08, 95\%Cl:1.06-1.10), being male (OR 4.41, 95\%Cl: 2.58-7.52), dyspnoea (OR 6.11, 95\%Cl: 3.58-10.45), dry cough (OR 2.89,95\%Cl: 1.54-5.41), wet cough (OR 4.62, 95\%Cl: 1.93-11.06), hypertension (OR 2.20, 95\%Cl: 1.17-4.16) and renal failure (OR 5.39, 95\%Cl: 1.00-29.00).

\section{Sensitivity analysis}

To examine if the discriminatory power of the predictive function changed as a function of data collected at different stages of the pandemic, a sensitivity analysis was performed. Accordingly, we compared the AUC of the model using the full dataset with the AUCs of models 
when sub-datasets were constructed by removing data from a specific stage (pre-lockdown, lockdown, and post-lockdown), socio-demographics, clinical symptoms, and comorbidities. The results showed that data collected from different stages of the pandemic or removing one significant variable at a time did not significantly alter the AUC of the ROC curve, as shown

Table 3. Multivariate logistic regression analysis regarding the probability of hospitalization vs. ambulance care among COVID-19 confirmed patients using wave 1 dataset (Model 1).

\begin{tabular}{lcccl}
\hline Variables & Coefficient & $\begin{array}{c}\text { Standard } \\
\text { error }\end{array}$ & $p$ Value & OR $(95 \% \mathrm{Cl})$ \\
\hline Socio-demographics & & & & \\
$\quad$ Age & 0.08 & 0.01 & $<.001$ & $1.08(1.06-1.10)$ \\
$\quad$ Gender - Male & 1.48 & 0.27 & $<.001$ & $4.41(2.58-7.52)$ \\
Clinical signs and comorbidities & & & & \\
$\quad$ Dyspnoea & 1.81 & 0.27 & $<.001$ & $6.11(3.58-10.45)$ \\
Rhinorrhea & -0.84 & 0.3 & .006 & $0.43(0.24-0.79)$ \\
Dry cough & 1.06 & 0.32 & .001 & $2.89(1.54-5.41)$ \\
Wet cough & 1.53 & 0.45 & .001 & $4.62(1.93-11.06)$ \\
Headache & -1.01 & 0.29 & .001 & $0.36(0.20-0.65)$ \\
Arterial hypertension & 0.79 & 0.32 & .015 & $2.2(1.17-4.16)$ \\
Chronic renal failure & 1.68 & 0.86 & .05 & $5.39(1.00-29.00)$ \\
Constant & -4.33 & 0.65 & & \\
\hline
\end{tabular}

${ }^{\mathrm{a}}$ Referenced category $=$ No; $R^{2}=0.504$ (Cox-Snell), 0.680 (Nagelkerke). Model $\chi^{2}(9)=400.491, p<.001$.

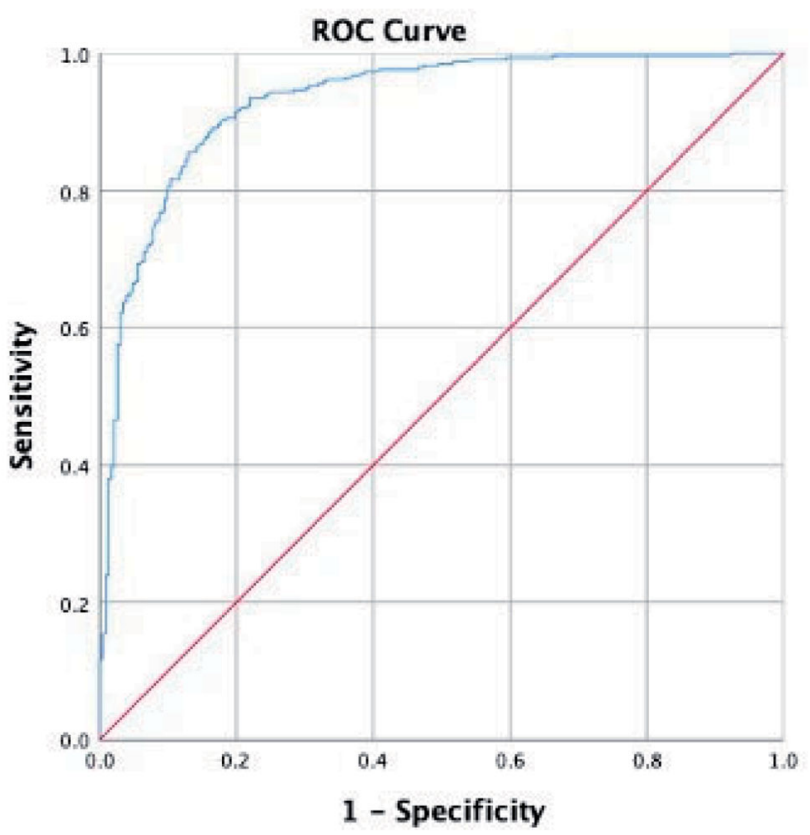

Figure 1. Area under curve (AUC) of the ROC curve $=0.931$ (95\% $\mathrm{Cl}$ : 0.910-0.953), standard error $(\mathrm{SE})=0.011$, using wave 1 dataset. by the overlapping $95 \% \mathrm{Cl}$ of the AUCs obtained (Table 4). Interestingly, the model performed better using the pre-lockdown and post-lockdown datasets. However, this difference was not significant.

In order to examine the effects of age and gender on the prediction model, we performed a multivariate regression analysis without these two socio-demographic variables and included all the significant factors from the univariate analysis. All significant predictors from the previous model upheld their predictive value (Table $A 1$ in Appendix), including myalgia ( $O R=0.54$, 95\% Cl: $0.31-0.93)$ and cardiac disease $(\mathrm{OR}=2.78$, 95\%Cl: 1.45-5.34). The model was calibrated (Hosmer-Lemeshow test $\left.\chi^{2}(8)=14.43, p=.071\right)$, and accounted for $R^{2}=0.394$ (Cox-Snell) and $R^{2}=0.532$ (Nagelkerke) with an AUC of 0.882 (95\% Cl: 0.854-0.911), and SE of 0.014. Employing fit measures including the Hosmer-Lemeshow test, the two $R^{2}$ values obtained, and the AUC, the model with age and gender proved to be more efficient in predicting hospitalization.

We tested if the model might be altered if variables with $p<.10, p<.15$, and $p<.20$ in the univariate analysis, namely anosmia $(p=.126)$ and diarrhoea $(p=.192)$ were added into the multivariate model. Adding anosmia did not change the predictive value of the relevant variables in Equation (1). However, diarrhoea was a significant predictor (OR 3.52, 95\%Cl: 1.80-6.88, $p<.001$ ) and changed the significance of renal failure ( $p$-value rose from .050 to .580). Retaining renal failure significantly reduced the -2 log-likelihood, and improved the overall model fit. Therefore, the variable was not removed from the model. The AUC (ROC) was 0.937 (0.916-0.957), and SE 0.010. The result of the multivariate analysis (Model 2) is shown in Table A2 in Appendix.

\section{Model validation}

To validate the predictive power of the prediction model, we applied Equation (1) to predict the level of care, i.e. ambulatory care and hospitalization for patients with confirmed COVID-19 in wave 2. Model 1 performed quite well as $82.2 \%$ of the cases were correctly classified with a sensitivity of $93.9 \%$ and a specificity of $67.1 \%$. Using model 2 with diarrhoea, $78.7 \%$ of the cases were

Table 4. Area under the receiver operating characteristic curve (AUC-ROC) in function of the dataset used.

\begin{tabular}{|c|c|c|}
\hline Dataset & AUC-ROC (95\% Cl) & Standard error (SE) \\
\hline All demographic characteristic and relevant symptoms and comorbidities included (as a reference) $=\mathrm{ALL}(N=571)$ & $0.931(0.910-0.953)$ & 0.011 \\
\hline ALL periods minus pre-lockdown $(n=515)$ & $0.925(0.900-0.949)$ & 0.012 \\
\hline ALL periods minus lockdown $(n=75)$ & $0.966(0.927-1.000)$ & 0.020 \\
\hline ALL periods minus post-lockdown $(n=552)$ & $0.931(0.909-0.953)$ & 0.011 \\
\hline
\end{tabular}


Table 5. Model comparison across model fit and performance criteria.

\begin{tabular}{|c|c|c|c|}
\hline Dataset & Criteria & Model 1 (9 independent variables) & Model 2 (10 independent variables) \\
\hline \multirow[t]{7}{*}{ Wave 1} & -2 Log-likelihood & 370.91 & 356.16 \\
\hline & Cox-Snell $R^{2}$ & 0.50 & 0.52 \\
\hline & Nagelkerke $R^{2}$ & 0.68 & 0.70 \\
\hline & Hosmer and Lemeshow Test & $\chi^{2}(8)=7.88, p=.44$ & $\chi^{2}(8)=7.726, p=.46$ \\
\hline & Sensitivity & $90.56 \%$ & $90.86 \%$ \\
\hline & Specificity & $81.47 \%$ & $81.47 \%$ \\
\hline & AUC-ROC & $0.931(0.910-0.953)$ & $0.937(0.916,0.957)$ \\
\hline \multirow[t]{3}{*}{ Wave 2} & Sensitivity & $93.88 \%$ & $92.86 \%$ \\
\hline & Specificity & $67.11 \%$ & $60.53 \%$ \\
\hline & AUC-ROC ${ }^{\mathrm{a}}$ & $0.913(0.870-0.954)$ & $0.909(0.863-0.951)$ \\
\hline
\end{tabular}

${ }^{\mathrm{a}} 95 \% \mathrm{Cl}$ of the AUC-ROC was obtained based on 2000 bootstrapped samples.

Table 6. Risk score models and the respective area under the receiver operating characteristic curve (AUC-ROC) with 95\% confidence interval.

\begin{tabular}{|c|c|c|c|c|}
\hline Variable & Type & Risk score Model 1 (points) & Risk score Model 2 (points) & Risk score Model 3 (points) \\
\hline Age & Risk & 3 & 5 & 1 \\
\hline Male & Risk & 14 & 12 & 10 \\
\hline Dyspnoea & Risk & 19 & 16 & 15 \\
\hline Rhinorrhea & Protection & 7 & 19 & 2 \\
\hline Dry cough & Risk & 9 & 7 & 8 \\
\hline Wet cough & Risk & 15 & 9 & 15 \\
\hline Headache & Protection & 9 & 23 & 2 \\
\hline Hypertension & Risk & 7 & 5 & 6 \\
\hline Renal failure & Risk & 17 & 4 & 41 \\
\hline \multicolumn{2}{|l|}{ Total points } & 100 & 100 & 100 \\
\hline \multicolumn{2}{|c|}{ AUC-ROC $(95 \% \mathrm{Cl})$ for wave 1 dataset } & $0.874(0.846-0.902)$ & $0.873(0.845-0.901)$ & $0.833(0.799-0.866)$ \\
\hline \multicolumn{2}{|c|}{ AUC-ROC $(95 \% \mathrm{Cl})$ for wave 2 dataset } & $0.863(0.808-0.918)$ & $0.833(0.774-0.893)$ & $0.859(0.804-0.915)$ \\
\hline
\end{tabular}

correctly classified, with a sensitivity of $92.9 \%$ and a specificity of $60.5 \%$. The AUC-ROC obtained from models 1 and 2 using bootstrapping on 2000 bootstrap samples was $0.913(95 \% \mathrm{Cl} \quad 0.870-0.954)$ and $0.909 \quad(95 \% \mathrm{Cl}$ $0.863-0.951)$, respectively.

\section{Model selection}

In order to select the final prediction model, different criteria were compared for model 1 and model 2 as presented in Table 5 . In the process of model building, adding diarrhoea resulted in a slightly better model fit, i.e. a small decrease in the -2 log-likelihood value, an increase of 0.02 in $R^{2}$, and $0.3 \%$ higher sensitivity. Applying the two models to predict the level of care of the 174 patients in wave 2 , model 1 outperformed model 2 with regard to sensitivity and specificity with a slightly higher AUC-ROC. Based on the criteria of model parsimony and validation results, model 1 was selected as the final model for discussion.

\section{Risk score models}

Based on the multivariate logistic regression model, three risk score models were developed based on the ORs (risk score Model 1), the lower and upper $95 \% \mathrm{Cl}$ of the ORs, namely risk score Model 2 and risk score Model 3 , respectively. Accordingly, the score for each independent risk or protection factor was assigned as an integer value that was close to the OR divided by the sum of all ORs in the model and summed up to 100 . Using Youden's index [12], the best cut-off for age was 54 years with $A U C=0.861(0.830-0.892)$, a sensitivity equal to $83.5 \%$ and a specificity of $76.3 \%$. Applying this cut-off for age, three risk score models were developed. To examine the relationship between the risk score and the outcome of hospitalization, we calculated the AUC for each risk score model and for each wave of data. The result is detailed in Table 6 , which shows that risk score model 1 based on the OR was the best one. Applying the Youden's index, the cut-off for the risk score model 1 is 41 points, with a sensitivity of $81.6 \%$ and specificity of $82.9 \%$.

\section{Discussion}

The present study found that old age, male gender, dyspnoea, dry cough, wet cough, hypertension, and renal failure were significant predictors of hospitalization in patients with confirmed COVID-19. Rhinorrhea and headache were protective factors associated with ambulatory care. 
Consistent with previous studies, old age and male gender were predictors of hospitalization in patients with confirmed COVID-19 [13]. Old age is associated with reduced immune-response [14]. On the other hand, female patients are, in general, more adaptive to immune responses, which results in higher immunity to viruses [15]. Therefore, the intensity of viral infection is lower in female patients. These factors may explain why older male patients are more likely to develop complications during the infection and at higher risk of hospitalization.

Dyspnoea, dry cough, and wet cough were associated with hospitalization, which is in accordance with a previous report [16] where the chest CT and clinical data of the patients were compared. Whereas fever, cough, and dyspnoea are identified as typical symptoms of COVID-19 [17], only dyspnoea was significantly associated with higher mortality as shown in the metaanalysis by Shi et al. [18]. On the other hand, dry cough and productive cough were symptoms observed in hospitalized patients [19]. Interestingly, rhinorrhea and headache, the two most frequently observed symptoms in patients with COVID-19, were protective factors for hospitalization.

Hypertension and renal failure were significant predictors of hospitalization. The results are in accordance with studies by Jakhmola et al. [20] and Richardson et al. [6], who found that hospitalized patients frequently presented with hypertension and kidney disease as well as with those of Ryan et al. [3] and Harrison et al. [21]. In contrast, despite significance in the univariate analysis, diabetes, cardiac disease, and cancer were not independent predictors of hospitalization in the multivariate analysis. This finding is either in line with or in contrast to previous studies [6,21]. The differing results might be explained by the heterogeneity of the data collected regarding comorbidity categories and COVID-19 severity in most studies. For instance, most studies did not pay attention to cancer [20].

The prediction model had a relatively large explained variance $\left(R^{2}=0.504\right.$ (Cox-Snell) and $R^{2}=$ 0.680 (Nagelkerke) and an AUC-ROC of 0.931, indicating that it was well calibrated. Using the predictive Equation (1), we achieved an AUC of 0.863 for the validation dataset. In both cases, the AUCs were higher than those obtained by Jehi et al. [13] who also employed age, gender, symptoms, and comorbidities to predict hospitalization in patients with COVID-19. Additionally, despite difference in the nature of the outcome, as Ryan et al. [3] also assessed patients who were admitted and then discharged as non-severe outcome, both studies found that clinical symptoms and comorbidities together with age and gender are important to adequately triage patients when they first arrive at the hospital.

The sensitivity analysis showed that the model had predictive power in all stages of the first wave of the pandemic. This finding is important in terms of model validation because it was supposed that there was greater demand for bed-capacity during the lockdown period when the infection curve was progressively reaching the peak. The addition of diarrhoea slightly altered the model, with renal failure becoming less significant. He et al. [22] found that both diarrhoea and chronic kidney disease were significant predictors of severe outcomes of hospitalized patients with COVID19. Therefore, the role of diarrhoea should not be neglected. Using the model with diarrhoea to predict the level of care in the wave 2 dataset, we obtained $3.4 \%$ fewer correctly classified cases and a slightly lower AUC-ROC. Thus, from a practical point of view and, taking into account the criterion of parsimony, the model presented in Equation (1) was more competitive, and, hence, is recommended as the final predictive model.

\section{Implications}

The predictive model presented in the present study might help resolve uncertainties in decisions about hospitalization of patients with confirmed COVID-19. The model might aid in early identification of patients whose life is threatened and should be hospitalized. It might, additionally, facilitate hospital bed management. Specific attention should be paid to older and male patients with dyspnoea, dry cough, wet cough, hypertension, and renal failure. Furthermore, the predictive model and alternatively the risk score model might be used in patient screening by general practitioners and could potentially reduce nosocomial transmission while ensuring an adequate level of care [3].

\section{Limitations}

Since the study was conducted at a single institution and used retrospective data, the findings should be interpreted with caution regarding their generalizability. It is acknowledged that the decision regarding 
hospitalization might depend on the availability of hospital beds. Therefore, when applying the model in another study, this concern should be taken into account. The small sample size with low prevalence of some comorbidities may have some consequence in the prediction model. Further research with larger sample sizes will provide more evidence to evaluate the predictive power. A multi-centre study collecting consistent data on socio-demographics, clinical symptoms, and comorbidities would contribute to a refined prognostic tool for the level of care in COVID-19 patients. Since patients under 16 years old were directed to a specialized ward, they were not included in the current study. However, so far, studies have shown that patients in this age group are less severely affected by COVID-19. Thus, the exclusion probably had a minimal impact on the model.

\section{Conclusion}

Employing age, gender, dyspnoea, rhinorrhoea, dry and wet cough, headache, and two comorbidities, namely hypertension and renal failure, we developed a model which might be used to triage patients to either ambulatory care or hospitalization. With a high AUC-ROC value in both the building and validation of the model and the risk score model, we are confident that this model can be useful in clinical decision-making not only in the Emergency Department but also in general practice. Problems with unnecessary crowding in hospitals might be mitigated, hence reducing the risk of the viral transmission and ensuring patient safety.

\section{Author contributions}

A.N.D., A.G., C.S., A.G., and A.F.D. designed the study. A.G., M.G., and V.D. were responsible for data collection and curation. A.N.D. performed the data analysis. All authors wrote and realized the final version of the manuscript.

\section{Disclosure statement}

The authors have no conflicts of interests.

\section{ORCID}

Anh Nguyet Diep (iD http://orcid.org/0000-0001-5134-3220

Anne-Françoise Donneau (D) http://orcid.org/0000-0002-5385-6289

\section{Data availability statement}

Anonymized data are available upon request.

\section{References}

[1] Johns Hopkins University. COVID-19 dashboard by the Center for Systems Science and Engineering (CSSE) at Johns Hopkins University (JHU). Johns Hopkins Coronavirus Resource Center (2020). [cited 2020 Dec 8]. Available from: https://coronavirus.jhu.edu/map.html

[2] de Best R. COVID-19 deaths worldwide per million population as of December 8, 2020, by country. 2020 [cited 2020 Dec 8]. Available from: https://www.statista.com/statistics/1104709/ coronavirus-deaths-worldwide-per-million-inhabitants/

[3] Ryan C, Minc A, Caceres J, et al. Predicting severe outcomes in Covid-19 related illness using only patient demographics, comorbidities and symptoms. Am J Emerg Med. 2020.

[4] Gilbert A, Brasseur E, Petit M, et al. Immersion in an emergency department triage center during the COVID-19 outbreak: first report of the Liège University Hospital experience. Acta Clin Belg. 2020..

[5] Clerkin KJ, Fried JA, Raikhelkar J, et al. COVID-19 and cardiovascular disease. Circulation. 2020;141:1648-1655.

[6] Richardson S, Hirsch JS, Narasimhan M, the Northwell COVID-19 Research Consortium, et al. Presenting characteristics, comorbidities, and outcomes among 5700 patients hospitalized with COVID-19 in the New York City area. JAMA. 2020;323:2052-2059.

[7] Su H, Yang M, Wan C, Yi LX, et al. Renal histopathological analysis of 26 postmortem findings of patients with COVID19 in China. Kidney Int. 2020;98:219-227.

[8] Wu Z, McGoogan JM. Characteristics of and important lessons from the coronavirus disease 2019 (COVID-19) outbreak in China: summary of a report of 72314 cases from the Chinese Center for Disease Control and Prevention. JAMA. 2020;323:1239-1242.

[9] Cheng $Y$, Luo R, Wang K, et al. Kidney disease is associated with in-hospital death of patients with COVID-19. Kidney Int. 2020;97:829-838.

[10] Gao J, Huang X, Gu H, et al. Predictive criteria of severe cases in COVID-19 patients of early stage: a retrospective observational study. J Clin Lab Anal. 2020;34: e23562.

[11] Wynants L, Van Calster B, Collins GS, et al. Prediction models for diagnosis and prognosis of covid-19 infection: systematic review and critical appraisal. BMJ. 2020;369: m1328.

[12] R Core Team. R: a language and environment for statistical computing. Vienna (Austria): $\mathrm{R}$ Foundation for Statistical Computing; 2013. Available from: http://www.Rproject.org/

[13] Jehi L, Ji X, Milinovich A, et al. Development and validation of a model for individualized prediction of hospitalization risk in 4,536 patients with COVID-19. PLoS One. 2020;15: e0237419. 
[14] Montecino-Rodriguez E, Berent-Maoz B, Dorshkind K. Causes, consequences, and reversal of immune system aging. J Clin Invest. 2013;123:958-965.

[15] Klein SL, Flanagan KL. Sex differences in immune responses. Nat Rev Immunol. 2016;16:626-638.

[16] Li K, Wu J, Wu F, et al. The clinical and chest CT features associated with severe and critical COVID-19 pneumonia. Invest Radiol. 2020;55:1-5.

[17] Chen X, Shan Y, Wen Y, et al. Mesenchymal stem cell therapy in severe COVID-19: a retrospective study of short-term treatment efficacy and side effects. J Infect. 2020;81: 647-679.

[18] Shi L, Wang $Y$, Wang $Y$, et al. Dyspnea rather than fever is a risk factor for predicting mortality in patients with COVID-19. J Infect. 2020;81:647-679.

[19] Rivera-Izquierdo $M$, del Carmen Valero-Ubierna M, RdelAmo JL, et al. Sociodemographic, clinical and laboratory factors on admission associated with COVID-19 mortality in hospitalized patients: a retrospective observational study. PLoS One. 2020;15:e0235107.

[20] Jakhmola S, Indari O, Baral B, et al. Comorbidity assessment is essential during COVID-19 treatment. Front Physiol. 2020; 11:1-7.

[21] Harrison SL, Fazio-Eynullayeva E, Lane DA, et al. Comorbidities associated with mortality in 31,461 adults with COVID-19 in the United States: a federated electronic medical record analysis. PLoS Med. 2020;17:e1003321.

[22] He F, Luo $Q$, Lei $M$, et al. Risk factors for severe cases of COVID-19: a retrospective cohort study. Aging. 2020;12: 15730-15740.

[23] Youden WJ. Index for rating diagnostic tests. Cancer. 1950; 3:32-35.

\section{Appendix}

Table A1. Multivariate analysis for the outcome hospitalization vs. ambulance care (excluding age and gender) (Model 2).

\begin{tabular}{lrccc}
\hline Variables & \multicolumn{1}{c}{$B$} & $\mathrm{SE}$ & $p$ Value & $\begin{array}{c}\text { OR } \\
(95 \% \mathrm{Cl})\end{array}$ \\
\hline Dyspnoea & 1.71 & 0.23 & .000 & $5.54(3.51-8.75)$ \\
Rhinorrhea & -1.09 & 0.26 & .000 & $0.34(0.2-0.56)$ \\
Dry_cough & 0.76 & 0.27 & .004 & $2.15(1.28-3.61)$ \\
Wet_cough & 1.16 & 0.37 & .001 & $3.2(1.56-6.57)$ \\
Headache & -1.18 & 0.29 & .000 & $0.31(0.18-0.54)$ \\
Myalgia & -0.62 & 0.28 & .025 & $0.54(0.31-0.93)$ \\
Hypertension & 1.53 & 0.29 & .000 & $4.61(2.64-8.06)$ \\
Cardiac_disease & 1.02 & 0.33 & .002 & $2.78(1.45-5.34)$ \\
Renal_failure & 1.58 & 0.78 & .044 & $4.86(1.05-22.61)$ \\
Constant & -0.46 & 0.25 & .069 & \\
\hline
\end{tabular}

${ }^{a}$ Referenced category $=$ No; $R^{2}=0.394$ (Cox-Snell), 0.532 (Nagelkerke). Model $\chi^{2}(8)=285.92, p<.001$.

Table A2. Multivariate logistic regression analysis for the level of care among COVID-19 confirmed patients using wave 1 dataset, including diarrhoea.

\begin{tabular}{lcccl}
\hline Variables & $B$ & SE & $p$ Value & OR $(95 \% \mathrm{Cl})$ \\
\hline Socio-demographics & & & & \\
$\quad$ Age & 0.08 & 0.01 & $<.001$ & $1.08(1.06,1.10)$ \\
$\quad$ Gender - Male & 1.64 & 0.29 & $<.001$ & $5.14(2.94,8.98)$ \\
Clinical signs and comorbidities $s^{\mathrm{a}}$ & & & \\
$\quad$ Dyspnoea & 1.83 & 0.28 & $<.001$ & $6.26(3.61,10.85)$ \\
Rhinorrhea & -1.02 & 0.32 & .001 & $0.36(0.19,0.67)$ \\
Dry_cough & 1.02 & 0.33 & .002 & $2.77(1.46,5.23)$ \\
Wet_cough & 1.51 & 0.45 & .001 & $4.55(1.87,11.04)$ \\
Diarrhoea & 1.26 & 0.34 & $<.001$ & $3.52(1.80,6.88)$ \\
Headache & -1.25 & 0.31 & .001 & $0.29(0.16,0.53)$ \\
Hypertension & 0.81 & 0.33 & .014 & $2.24(1.18,4.25)$ \\
Renal_failure & 1.67 & 0.88 & .058 & $5.30(0.95,29.61)$ \\
Constant & -6.02 & 0.70 & &
\end{tabular}

${ }^{a}$ Referenced category $=$ No; $R^{2}=0.517$ (Cox-Snell), 0.697 (Nagelkerke). Model $\chi^{2}(10)=415.249, p<.001$. 